\title{
A Table of Totally Real Cubic Fields
}

\author{
By I. O. Angell
}

\begin{abstract}
In this paper the author describes the construction of a table of totally real cubic number fields. Each field is distinguished by the coefficients of a generating polynomial, the index of this polynomial over the field and the discriminant of the field. The class number and a fundamental pair of units is also given.
\end{abstract}

This paper describes the construction of a table of the 4794 totally real nonconjugate cubic fields with discriminants less than 100,000, which extends the existing table of fields with discriminants less than 20,000 given by Godwin and Samet [7] . It was discovered that two fields are missing from the latter table, namely those with discriminants 13549,19736 . Both have class number 1 .

The table complements the author's existing table of the 3169 complex nonconjugate cubic fields with discriminants greater than $-20,000$ (Angell [1], and Shanks [8]). It has been deposited with the Mathematics of Computation's Depository of Unpublished Mathematical Tables; copies may also be obtained from the author on request.

Initially, a table of polynomials was produced using the method developed by Godwin [6] which shows that a real cubic field with discriminant $\Delta$, and its conjugates, are generated by the zeros $\alpha, \beta, \gamma$ of a polynomial $f(x)=x^{3}-a x^{2}+b x-c$ where

$$
S=1 / 2\left\{(\alpha-\beta)^{2}+(\beta-\gamma)^{2}+(\gamma-\alpha)^{2}\right\}=a^{2}-3 b \leqslant \Delta^{1 / 2} .
$$

By stipulating that the zeros may be constrained to the bounds

$$
0 \leqslant \alpha<1, \quad \alpha \leqslant \beta \leqslant \gamma, \quad \alpha+\gamma \leqslant 2 \beta,
$$

the following inequalities were produced

$$
\begin{gathered}
1 \leqslant a<3+2 \Delta^{1 / 4}, \\
2 a^{2} / 9 \leqslant b \leqslant a^{2} / 3, \\
1 \leqslant c \leqslant \min \left(b-a+1,\left(9 a b-2 a^{3}\right) / 27\right) .
\end{gathered}
$$

Setting $\Delta=100,000$, then a list including all of the polynomials whose zeros generate all the real cubic number fields with discriminant less than 100,000 was calculated. This list included superfluous polynomials, i.e. those which were reducible or had negative discriminants. Each remaining polynomial, with discriminant $D>0$, generated three conjugate real cubic fields (or a single cyclic field) with field discriminant $\Delta=D / k^{2}$ where $k^{2}$ divides $D$. Since $D \leqslant 4 S^{3} / 27$ and $S^{2} \leqslant 100,000$, then

$$
k^{2} \leqslant D / S^{2} \leqslant 4 S / 27 \leqslant 4(100,000)^{1 / 2} / 27 \text {, }
$$

whence $k \leqslant 6$.

Received March 26, 1975 ; revised July 1, 1975.

AMS (MOS) subject classifications (1970). Primary 12-04, 12A30, 12A45, 12 A50. 
Thus, any polynomial in the list with index greater than 6 was ignored because the list had to include another polynomial which generated the same field as the former.

Each polynomial with zero $\theta$ generates a field $K=Q(\theta)$; and to find $\Delta$, the discriminant of $K$, it was necessary to examine all the squared factors of the polynomial discriminant $D$. Initially, $k^{\prime}$ was found, so that $D / k^{\prime 2}$ was square free; and then individual factors of $k^{\prime}$ were tested to see if they divided $k$. This was done in two stages:

(i) Bachmann's method [2] showed whether $k$ was divisible by a prime divisor $p$ of $k^{\prime}$.

(ii) If $k$ was divisible by such a prime $p$ and furthermore $k^{\prime}$ was divisible by $p^{2}$, then all numbers of the form

$$
\left(\theta^{2}+f \theta+g\right) / p \quad \text { where } 0 \leqslant f, g \leqslant p-1,
$$

were checked to see if they were algebraic integers.

If $k$ was divisible by 4 and $k^{\prime}$ divisible by 8 , then all numbers of the form

$$
(\theta+g) / 2, \quad g=0 \text { or } 1,
$$

were also checked to see if they were algebraic integers. Since the smallest discriminant is 49 , then the largest prime $p$ needing consideration was $\leqslant \sqrt{\Delta / 49} \leqslant \sqrt{100,000 / 49}$, hence $p \leqslant 43$.

It still remained to test whether fields of the same discriminant were the same field. This was achieved by finding a Tschimhausen transformation between the two polynomials, a matter of solving three equations in three unknowns (the coefficients of the transformation); the solutions were rational integers if and only if the polynomials produced the same field.

The completed table also contains the class number and a fundamental pair of units for each field. These additional entries were furnished by Voronoi's algorithm (Delone and Faddeev [5]). Given any ideal in the field, this algorithm produces a finite network of ideals, such that two ideals produce the same network if and only if they are equivalent. Hence the number of different networks in a field is the class number of that field. Therefore, to find the class number of a field, all the ideals with norm less than the Minkowski-Davenport bound $\Delta^{1 / 2} / 7$ (Davenport [3]) were calculated and stored in a list, accessible by a pointer table. Progressing through this list, the network emanating from each ideal was produced by Voronoi's algorithm. If it was discovered that the network included an ideal that had been calculated previously, then the uniqueness of a network class implied that the network under construction had been found earlier, and so the procedure was terminated and the next ideal in the list considered. Also, every ideal produced by this algorithm need not be used to initialize a further network, whence every newly constructed ideal was deleted from the original list. A unique class structure of networks was finally compiled, giving the class number of the field.

The network originating from the unit ideal (hence all ideals in this network are principal) also provided a fundamental pair of units. The algorithm specifies two subsets of the principal ideals, and when all the individual elements in each subset are multiplied together, the units are produced. 
The table supports the bound produced by Davenport and Heilbronn [4] for the density of real cubic fields with discriminants less than 100,000 .

Statistics referring to the class numbers in the table are given below.

\begin{tabular}{|c|c|c|c|c|c|c|c|c|c|c|}
\cline { 3 - 10 } & \multicolumn{7}{|c|}{ Class Number } \\
\hline Bounds on $\Delta$ & $\begin{array}{c}\text { Total number } \\
\text { of fields }\end{array}$ & 1 & 2 & 3 & 4 & 5 & 6 & 7 & 8 & 9 \\
\hline 10001 to 10000 & 382 & 358 & 9 & 14 & 1 & - & - & - & - & - \\
\hline 20001 to 30000 & 466 & 414 & 26 & 21 & 2 & 2 & - & 1 & - & - \\
\hline 30001 to 40000 & 471 & 418 & 23 & 24 & 2 & 4 & - & - & - & - \\
\hline 40001 to 50000 & 485 & 418 & 29 & 33 & 3 & 1 & 1 & - & - & - \\
\hline 50001 to 60000 & 500 & 442 & 27 & 23 & 1 & 1 & 2 & 3 & 1 & - \\
\hline 60001 to 70000 & 490 & 417 & 32 & 33 & 3 & 4 & - & - & - & 1 \\
\hline 70001 to 80000 & 509 & 436 & 35 & 30 & 3 & 3 & 2 & - & - & - \\
\hline 80001 to 90000 & 514 & 432 & 44 & 33 & 2 & 2 & - & 1 & - & - \\
\hline 90001 to 100000 & 527 & 441 & 42 & 37 & 1 & 2 & 2 & 2 & - & - \\
\hline
\end{tabular}

Of these 4794 fields, except for the field discriminants listed below, all discriminants have only one nonconjugate field.

Discriminants with two associated nonconjugate fields:

$3969,8281,13689,17689,29241,37300,38612,45684,46548,47089$,

55700, 61009, 66825, 67081, 69012, 77841, 83700, 90601, 92340.

Discriminants with three associated nonconjugate fields:

22356, 28212, 31425, 41332, 47860, 54324, 57588, 58077, 62004, 62644, 63028, 65908, 77844, 82484, 86485, 86828, 89073, 95992, 97844, 98132, 99860.

Discriminants with four associated nonconjugate fields:

$$
32009,42817,62501,72329,94636 .
$$

The FORTRAN IV programs used in compiling this table were run on the University of London's CDC 6600 machine. The total running time was thirty-two min- 
utes. I wish to thank Professor H. J. Godwin and Dr. D. Shanks for their invaluable advice and assistance during the production of this table.

Department of Statistics and Computer Science

Royal Holloway College

University of London

London, England

1. I. O. ANGELL, "A table of complex cubic fields," Bull. London Math. Soc., v. 5, 1973, pp. 37-38. MR 47 \#6648.

2. P. BACHMANN, Algemeine Arithmetik der Zahlkörper, Leipzig, 1905.

3. H. DAVENPORT, "On the product of three homogeneous linear forms. II," Proc. London Math. Soc. (2), v. 44, 1938; pp. 412-431.

4. H. DAVENPORT \& H. HEILBRONN, "On the density of discriminants of cubic fields," Bull. London Math. Soc., v. 1, 1969, pp. 345-348. MR 40 \#7223.

5. B. N. DELONE \& D. K. FADDEEV, The Theory of Irrationalities of the Third Degree, Trudy Mat. Inst. Steklov., v. 11, 1940; English transl., Transl. Math. Monographs, vol. 10, Amer. Math. Soc., Providence, R. I., 1964. MR 2, 349; 28 \#3955.

6. H. J. GODWIN, "On totally complex quartic fields with small discriminants," Proc. Cambridge Philos. Soc., v. 53, 1957, pp. 1-4. MR 18, 565.

7. H. J. GODWIN \& P. A. SAMET, "A table of real cubic fields," J. London Math. Soc., v. 34, 1959, pp. 108-110. MR 20 \#7009.

8. D. SHANKS, “Review of I. O. Angell, A table of complex cubic fields," Math. Comp.,v. 29, 1975, pp. 661-665. RMT 33. 